\title{
The Chemotactic Response of Plasmodia of the Myxomycete Physarum polycephalum to Sugars and Related Compounds
}

\author{
By DAVID J. C. KNOWLES* AND MICHAEL J. CARLILE \\ Department of Biochemistry, Imperial College of Science and Technology, \\ London SW7 $2 A Z$
}

(Received 15 March 1978)

\begin{abstract}
A new technique, the double strip method, for studying the chemotaxis of myxomycete plasmodia is described. Physarum polycephalum was attracted by the aldohexoses D-glucose, $\mathrm{D}$-galactose and D-mannose and their derivatives 2-deoxy-D-glucose and maltose, thresholds ranging from $0.25 \mathrm{~mm}$ (D-glucose) to $5 \mathrm{~mm}$ (D-mannose). These sugars competed with each other, a uniform background of one of them inhibiting taxis to the others. Other attractants were $N$-acetyl-D-glucosamine and mannitol, with thresholds at $1 \mathrm{~mm}$, and fucose (6-deoxy-D-galactose). Although in general only those carbohydrates which could support growth were attractants, there were exceptions such as 2-deoxy-D-glucose; hence metabolism of a compound was not necessary for attraction. In addition, some compounds, such as fructose, could be metabolized but did not attract. At high concentrations (about $100 \mathrm{~mm}$ ) all the compounds tested, including attractants, could under appropriate conditions cause repulsion, probably through osmotic effects.
\end{abstract}

\section{INTRODUCTION}

Carlile (1970) found that plasmodia of the myxomycete Physarum polycephalum Schweinitz were attracted by (i.e. showed positive chemotaxis to) glucose, maltose, mannose and galactose but not by sucrose, fructose or ribose. Each of the former group of sugars supported growth in shaken liquid culture when present as the sole carbohydrate, but the latter group did not. It was tentatively concluded that with $P$. polycephalum there was a parallel between the chemotactic effectiveness of sugars and their ability to support growth.

However, studies on chemotaxis with Escherichia coli have established that although most nutrients are attractants, some compounds are metabolized but do not attract, and others are not metabolized but are strong attractants (Adler, 1975). This conclusion suggests that the chemotactic responses of $P$. polycephalum to a wider range of sugars should be examined, and compared with the ability of the sugars to support growth and influence migration. Studies on growth and migration were reported in the preceding paper (Knowles \& Carlile, 1978); studies on chemotaxis are described here.

* Present address: Department of Experimental Chemotherapy, May \& Baker Research Institute, Dagenham, Essex. 

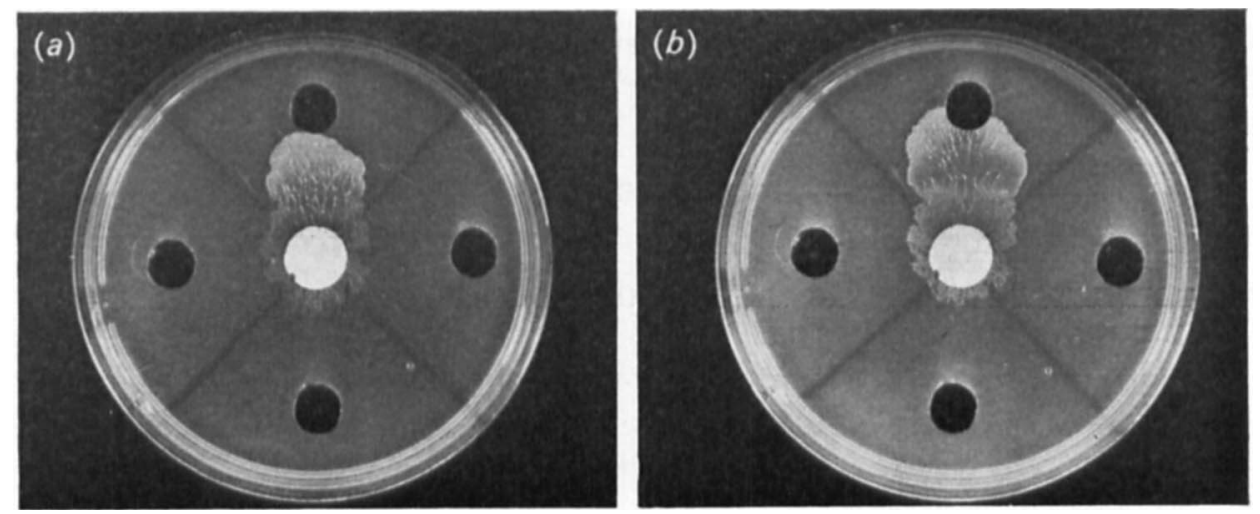

Fig. 1. Quadrant test, showing the response of a plasmodium on SVP agar to SVP plus $100 \mathrm{mm-}$ glucose solution in the top well: $(a) 15 \frac{1}{2} \mathrm{~h}$ and $(b) 20 \mathrm{~h}$ after inoculation. The morphology of the plasmodium indicates that an approximately uniform spreading has been followed by oriented movement towards the test well when the attractant was encountered.

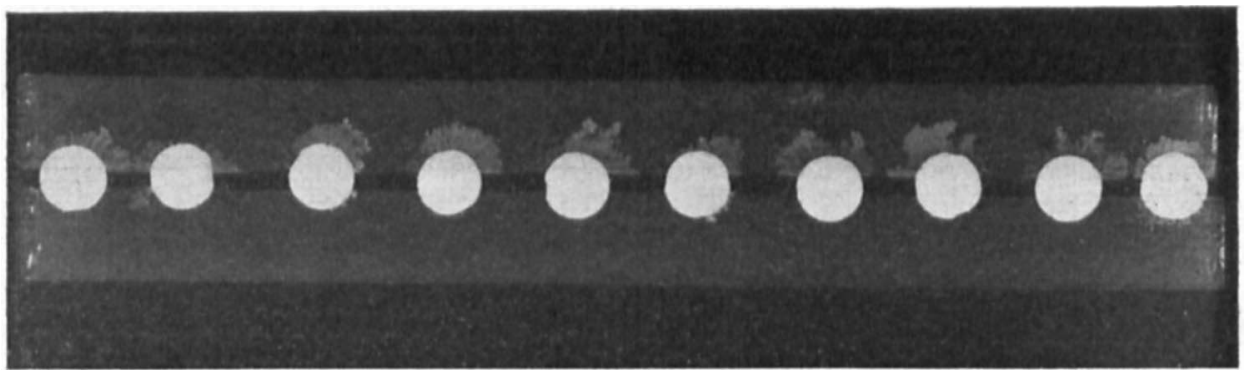

Fig. 2. Double strip test showing the response of plasmodia at $4 \mathrm{~h}$ to salts agar plus $10 \mathrm{~mm}$-glucose; the lower, control strip contained salts agar only.

\section{METHODS}

Strain, media and routine culture. Strain i $\times$ A7029 (Carlile, 1972) was maintained in shaken liquid culture on SVP $1 \%$ glucose medium which contains salts, vitamins, peptone and glucose (Knowles \& Carlile, 1978). Inocula for experiments were obtained from plasmodia ( 3 to $5 \mathrm{~d}$-old and 100 to $200 \mathrm{~mm}$ diam.) growing on $5 \mathrm{~mm}$ deep SVP $1 \%$ starch agar in $250 \times 250 \mathrm{~mm}$ bioassay dishes by placing 11 or $13 \mathrm{~mm}$ diam. discs cut from cellulose nitrate membranes (Schleicher and Schuell BA83; Andermann \& Co., East Molesey, Surrey) around the edge of the plasmodia. The membranes were covered by the advancing plasmodium and could be lifted off easily. Routine culture and experiments were carried out at $24^{\circ} \mathrm{C}$ and aseptic methods were employed except where otherwise stated.

The quadrant test for chemotaxis. The procedure was based on that of Madelin et al. (1975). Petri dishes (90 $\mathrm{mm}$ diam.) containing agar medium were marked into quadrants and a $9 \mathrm{~mm}$ diam. well was cut in each quadrant (Fig. 1). Into one well was placed $0.25 \mathrm{ml}$ of a filter-sterilized test solution and into the other wells were placed portions of a control solution corresponding to the agar medium. Petri dishes were centrally inoculated with plasmodia on $13 \mathrm{~mm}$ diam. cellulose nitrate membranes. Plates were incubated in darkness and scored as positive when the test well was the first to be reached by a plasmodium, recording being carried out at 15 and $20 \mathrm{~h}$. Replication was used and the probability of the result being due to random migration was assessed statistically (Madelin et al., 1975). When this probability was less than $1 \%$, chemotaxis was regarded as established. Probabilities $(P)$ from 1 to $5 \%$ were regarded as indicating probable chemotaxis. In experiments with a range of attractant or repellent concentrations, the lowest concentration giving $P$ less than $5 \%$ was taken as the threshold for the response. The quadrant test is a modification of the two-well test (Carlile, 1970); it is more efficient than the two-well test (i.e. gives results of comparable significance with fewer replicates) for establishing positive chemotaxis (complete success with four replicates gives a significantly positive result) but less effective with negative chemotaxis (at least 20 replicates needed).

The double strip test for chemotaxis. Strips of agar ( $200 \mathrm{~mm}$ long $\times 16 \mathrm{~mm}$ wide $\times 5 \mathrm{~mm}$ deep) were prepared 
Table 1. Response of plasmodia to different glucose concentrations in the presence of SVP agar in the quadrant test

Initial glucose
concn in
well $(\mathrm{mm})$
100
10
5
$2 \cdot 5$
1
$0 \cdot 75$
$0 \cdot 5$
$0 \cdot 25$
0

No. of
plasmodia
scored
10
20
20
10
20
20
20
10

$\begin{array}{cc}\begin{array}{c}\text { No. of } \\ \text { positive } \\ \text { responses }\end{array} & \text { Result* } \\ 10 & + \\ 20 & + \\ 19 & + \\ 10 & + \\ 14 & + \\ 13 & + \\ 9 & ++ \\ 2 & 0 \\ 1 & 0\end{array}$

$*+$, Attraction, $P$ (the probability of the result being due to random migration) $<1 \% ;(+)$, probable attraction, $P 1$ to $5 \%$; 0 , random migration, $P>5 \% ;(-)$, probable repulsion, $P 1$ to $5 \%$, , repulsion, $P<1 \%$. Statistical procedures from Madelin et al. (1975).

by pouring molten agar medium into plastic moulds. A strip containing the substance to be tested was then put on a glass plate $3 \mathrm{~mm}$ from a control strip lacking the test substance (Fig. 2). Ten plasmodia on $11 \mathrm{~mm}$ diam. cellulose nitrate discs were removed from SVP $1 \%$ starch agar, washed by floating on salts solution (containing the salts of SVP medium) for $5 \mathrm{~min}$, blotted on the under surface of the disc and placed so as to bridge the gap between the two agar strips. The plasmodia were incubated in a moist chamber in the dark and the number of plasmodia migrating on to the test and control strips were recorded, usually at $4 \mathrm{~h}$. Since tests were concluded so soon, strictly aseptic conditions were not considered necessary. Plasmodia moving from the disc were scored as positive if they moved on to the test strip, as negative if they moved on to the control strip, and as two plasmodia, one positive and one negative, if they spread on to both strips. The probability of the result obtained being due to random migration was assessed by means of the binomial distribution (Snedecor \& Cochran, 1974), and the values of $P$ obtained were interpreted as in the quadrant test. The double strip test detects attraction and repulsion with equal efficiency, and is simple and rapid, permitting one worker to test at least 500 plasmodia in a week as against about 150 with the quadrant test.

\section{RESULTS}

\section{Chemotactic responses in the quadrant test}

The response of plasmodia to different concentrations of D-glucose was tested on SVP agar (Table 1). Positive chemotaxis was observed at all concentrations tested above a threshold at $0.5 \mathrm{~mm}$. Various sugars were then tested on SVP agar at $100 \mathrm{~mm}$ and, in some instances, at $10 \mathrm{~mm}$ also (Table 2). The conclusion of Carlile (1970) that D-glucose, D-mannose, D-galactose and maltose were attractants, and that D-ribose and sucrose were not, was confirmed, and attraction to $\mathrm{N}$-acetyl-D-glucosamine and mannitol was also found. A positive response was observed with D-fructose, contrary to the earlier study. However, since this was seen only at $100 \mathrm{~mm}$ and was not observed in subsequent tests, and since the stock fructose contained about $0.1 \%$ glucose and perhaps other contaminants, it is probable that fructose is not an attractant. All the compounds, except D-mannosamine hydrochloride and $\mathrm{N}$-acetyl-D-galactosamine, were retested on water agar. Attraction was observed with glucose and maltose, although the proportion of positive results obtained was less than on SVP agar. All the other compounds tested failed to attract.

\section{Chemotactic responses in the double strip test}

The double strip test was used to examine the chemotactic responses of a range of compounds on a non-nutrient agar. The agar used contained the salts from SVP medium, since recovery of plasmodia transferred from SVP $1 \%$ starch agar to this salts agar was much 
Table 2. Response of plasmodia to various compounds on SVP agar in the quadrant test

\begin{tabular}{|c|c|c|}
\hline \multirow{2}{*}{ Compound* } & \multicolumn{2}{|c|}{ Result $\uparrow$} \\
\hline & $10 \mathrm{~mm}$ & $100 \mathrm{~mm}$ \\
\hline \multicolumn{3}{|l|}{ Pentoses } \\
\hline D-Ribose & NT & 0 \\
\hline D-Arabinose & NT & 0 \\
\hline L-Arabinose & NT & + \\
\hline \multicolumn{3}{|l|}{ Aldohexoses } \\
\hline D-Glucose & + & + \\
\hline D-Mannose & + & + \\
\hline D-Galactose & + & + \\
\hline \multicolumn{3}{|l|}{ Ketohexoses } \\
\hline D-Fructose & 0 & + \\
\hline L-Sorbose & NT & 0 \\
\hline \multicolumn{3}{|l|}{ Disaccharides } \\
\hline Maltose & + & + \\
\hline Sucrose & 0 & $(-)$ \\
\hline \multicolumn{3}{|l|}{ Hexosamines } \\
\hline D-Mannosamine. $\mathrm{HCl}$ & 0 & - \\
\hline$N$-Acetyl-D-glucosamine & + & + \\
\hline$N$-Acetyl-D-galactosamine & NT & 0 \\
\hline \multicolumn{3}{|l|}{ Sugar alcohol } \\
\hline Mannitol & + & + \\
\hline \multicolumn{3}{|l|}{ Deoxysugars } \\
\hline 2-Deoxy-D-glucose & 0 & 0 \\
\hline D-Fucose (6-deoxy-D-galactose) & NT & $(-)$ \\
\hline \multicolumn{3}{|l|}{ Alkyl glycoside } \\
\hline Methyl- $\alpha$-D-glucoside & 0 & 0 \\
\hline
\end{tabular}

* Concentrations of contaminating glucose $(\%)$ in stock chemicals were: D-ribose, 0.03 ; L-arabinose, $0.18 ;$ D-fructose, $0.12 ; \mathrm{L}$-sorbose, $0.01 ; N$-acetyl-D-glucosamine, $0.04 ; 2$-deoxy-D-glucose, 0.5 to $1 ;$ methyl- $\alpha$ D-glucoside, $0 \cdot 55$. The result for 2-deoxyglucose was obtained by gas-liquid chromatography of the silyl derivative (Sweeley et al., 1963); the others are from Knowles \& Carlile (1978).

$\dagger$ Symbols and statistical method as for Table 1; NT, not tested.

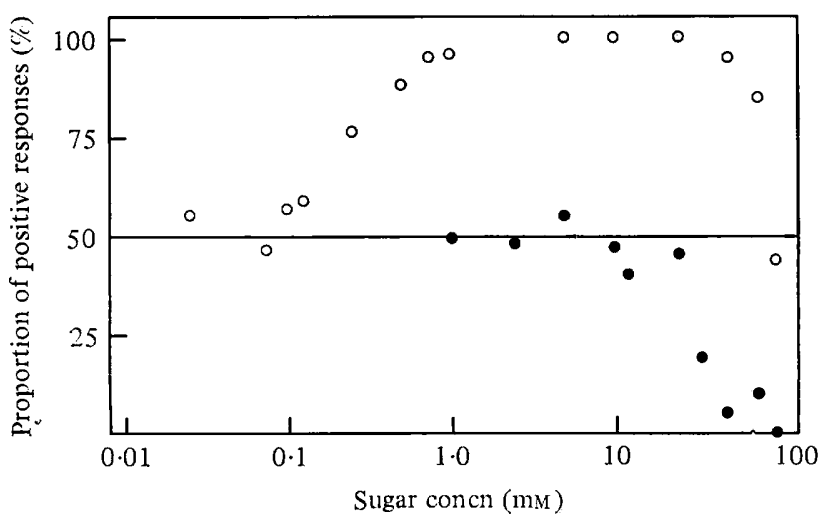

Fig. 3. Response of plasmodia to glucose $(\bigcirc)$ and to fructose $(\bullet)$ in the double strip test. Positive response by half the plasmodia indicates the absence of chemotaxis, a significantly higher proportion indicates attraction, and a significantly lower proportion indicates repulsion. It appears that glucose attracts over a wide range of concentrations, and that fructose repels at high concentrations. Each point is based on results with at least 10 plasmodia, although usually more (on average, 30 ) were employed. 
Table 3. Response of plasmodia to various compounds on salts agar in the double strip test

Inocula were from SVP $1 \%$ starch agar, except for experiments with mannitol, which failed to attract when inocula were from this medium, but did so when inocula were taken from SVP $1 \%$ mannitol agar.

Compound*
Pentoses
D-Ribose
D-Arabinose
L-Arabinose
Aldohexoses
D-Glucose
D-Galactose
D-Mannose
Ketohexoses
D-Fructose
L-Sorbose
Disaccharides
Maltose
Sucrose
Hexosamines
D-Glucosamine. HCl
D-Galactosamine. HCl
D-Mannosamine. HCl
$N$-Acetyl-D-glucosamine
$N$-Acetyl-D-galactosamine
$N$-Acetyl-D-mannosamine
Sugar alcohol
Mannitol
Deoxysugars
2-Deoxy-D-glucose
D-Fucose (6-deoxy-D-galactose)
Alkyl glycoside
Methyl- $\alpha$-D-glucoside
Detal

\begin{tabular}{|c|c|c|c|}
\hline \multicolumn{2}{|c|}{ Result $\dagger$} & \multicolumn{2}{|c|}{ Threshold (mM) } \\
\hline $10 \mathrm{~mm}$ & $100 \mathrm{~mm}$ & Attraction & Repulsion \\
\hline 0 & - & & 50 \\
\hline 0 & - & & 25 \\
\hline 0 & $0 \ddagger$ & & \\
\hline+ & 0 & $0 \cdot 25$ & \\
\hline+ & - & $0 \cdot 5$ & 100 \\
\hline+ & - & 5 & 75 \\
\hline 0 & - & & 35 \\
\hline 0 & - & & 25 \\
\hline+ & - & $0 \cdot 5$ & 75 \\
\hline 0 & - & & 25 \\
\hline- & - & & 5 \\
\hline- & NT & & \\
\hline- & NT & & 10 \\
\hline+ & - & 1 & 75 \\
\hline 0 & - & & 50 \\
\hline 0 & - & & 50 \\
\hline+ & - & 1 & \\
\hline+ & - & 0.5 & 100 \\
\hline+ & - & & \\
\hline 0 & - & & 100 \\
\hline
\end{tabular}

* For concentrations of contaminating glucose, see Table 2.

$\uparrow$ Symbols and statistical method as for Table 1; NT, not tested. Where a sufficient range of concentrations was tested the threshold for attraction, repulsion or both is indicated.

$\ddagger$ When strips were left for $15 \mathrm{~h}$, positive chemotaxis to L-arabinose was observed at concentrations of $25 \mathrm{~mm}$ and above. Because of the long delay in response, the high threshold and the relative impurity of the sugar (Table 2), it is unlikely that L-arabinose is an attractant.

more rapid than on water agar, migration beginning within 1 or $2 \mathrm{~h}$ and the experiment being complete in $4 \mathrm{~h}$. The results of the test with D-glucose and D-fructose are given in Fig. 3. Positive chemotaxis to glucose occurred from a threshold concentration of $0.25 \mathrm{~mm}$ and continued up to $75 \mathrm{~mm}$, but failed at $100 \mathrm{~mm}$, the highest concentration tested. No positive chemotaxis was observed with $D$-fructose, but significant repulsion occurred at concentrations of $35 \mathrm{~mm}$ and above. These results and those for the other compounds tested are summarized in Table 3. Attraction by D-galactose, D-mannose, maltose and $\mathrm{N}$-acetyl-D-glucosamine was confirmed, and approximate thresholds were determined. Attraction to mannitol did not occur with inocula from SVP 1\% starch agar, but took place with plasmodia from SVP $1 \%$ mannitol agar. Positive chemotaxis occurred to two compounds, 2-deoxy-D-glucose and 6-deoxy-D-galactose (fucose), which failed to attract in the quadrant test. All compounds except D-glucose and L-arabinose were repellent at $100 \mathrm{~mm}$.

Experiments were repeated with SVP agar instead of salts agar as the substratum. The only important differences observed were that for repulsion the thresholds were lower, and 
Table 4. Attraction to sugars and derivatives in the presence of a background of the same or other sugars and derivatives

Experiments were carried out on salts agar by the double strip test as in Table 3, except that both stiips contained a background sugar (apart from the control).

Attractant $\dagger$

$$
\begin{aligned}
& \text { Background } \\
& \text { sugar* }
\end{aligned}
$$

None (control)

D-Glucose

D-Galactose

D-Mannose

Maltose

2-Deoxy-D-

glucose

$N$-Acetyl-D-

glucosamine

\begin{tabular}{|c|c|c|c|c|c|}
\hline D-Glucose & D-Galactose & D-Mannose & Maltose & $\begin{array}{l}\text { 2-Deoxy- } \\
\text { D-glucose }\end{array}$ & $\begin{array}{l}N \text {-Acetyl-D- } \\
\text { glucosamine }\end{array}$ \\
\hline
\end{tabular}

Mannitol

$\begin{array}{ccc}+ & + & + \\ 0 & 0 & 0 \\ 0 & 0 & 0 \\ 0 & 0 & 0 \\ 0 & 0 & 0 \\ 0 & \text { NT } & \text { NT } \\ + & + & + \\ + & + & (+)\end{array}$

$\begin{array}{cccc}+ & + & + & + \\ 0 & 0 & (+) & 0 \\ 0 & 0 & + & 0 \\ 0 & 0 & + & 0 \\ 0 & 0 & + & 0 \\ \text { NT } & \text { NT } & + & \text { NT } \\ + & + & 0 & 0 \\ + & \text { NT } & + & 0\end{array}$

* 2-Deoxy-D-glucose was used at $10 \mathrm{~mm}$ and all other compounds at $50 \mathrm{~mm}$.

$\uparrow$ Glucose and maltose were used at $1 \mathrm{~mm}, 2$-deoxy-D-glucose at $2.5 \mathrm{~mm}$ and all other compounds at $5 \mathrm{~mm}$. Symbols and statistical method as for Table 1; NT, not tested.

\$ Experiments with a background of 2-deoxy-D-glucose were assessed at $15 \mathrm{~h}$, because migration was delayed.

that D-glucose and L-arabinose, which had not shown repulsion on salts agar, both showed significant repulsion within $6 \mathrm{~h}$ on SVP agar. The only threshold for positive chemotaxis which was accurately determined on SVP agar, that for D-glucose, was the same as that observed on salts agar, $0.25 \mathrm{~mm}$.

\section{Competition experiments}

Salts agar containing various sugars and derivatives was used to examine the effect of a uniform background of these compounds on the performance of attractants in the double strip test (Table 4). Controls in which the background was lacking showed normal positive chemotaxis and those in which the attractant under test was present in both strips showed random migration. Attraction by D-glucose was inhibited by uniform backgrounds of D-galactose, D-mannose, maltose, and 2-deoxy-D-glucose. No other compound tested, including the attractants $N$-acetyl-D-glucosamine and mannitol, interfered with positive chemotaxis to D-glucose. Tests on D-galactose, D-mannose, maltose and 2-deoxy-D-glucose indicated that their ability to attract was prevented by uniform backgrounds of any one of these four sugars and by glucose, but not by $\mathrm{N}$-acetyl-D-glucosamine or mannitol. Positive chemotaxis to $N$-acetyl-D-glucosamine was not inhibited by any of the other compounds, but attraction to mannitol was inhibited by all metabolizable sugars tested. The above competition experiments indicate that the attractants fall into three classes: D-glucose, D-galactose, D-mannose, maltose and 2-deoxy-D-glucose, which compete with each other; $N$-acetyl-D-glucosamine, which does not compete with and is not inhibited by any other attractant; and mannitol, which does not interfere with the response to other attractants although its action is prevented by attractants of the other two classes.

\section{DISCUSSION}

\section{Positive chemotaxis}

It is clearly valuable to an organism if sugars that support growth also act as attractants. A comparison of the present results on the chemotactic effectiveness of sugars with their ability to support growth (Knowles \& Carlile, 1978) indicates that there is a close parallel between utilization and attractiveness. Glucose, galactose, mannose, maltose and 
mannitol can all support growth as the sole carbohydrate, and all are attractants. Sucrose, sorbose and methyl- $\alpha$-D-glucoside cannot support growth and do not attract. However, although in general there is a parallel between the ability of a compound to support growth and to attract, there are exceptions which establish that, as with bacterial chemotaxis (Adler, 1975), metabolism is neither necessary nor sufficient for attraction. For example, ribose and fructose can support growth on agar, yet do not attract, whereas 2-deoxy-D-glucose is an attractant which is not metabolized and does not support growth (Knowles \& Carlile, 1978). Hence there is not a causal relationship between promotion of growth and chemotaxis.

Competition between attractants has been employed in studies on bacterial chemotaxis in order to determine how many types of chemoreceptors are involved (Adler, 1975). The present study showed competition between glucose, galactose, mannose, maltose and 2-deoxy-D-glucose; it is unlikely that there is a single chemoreceptor for all these sugars. Strange \& Koshland (1976) have, however, shown that in chemotaxis of Salmonella typhimurium competition occurs between galactose and ribose even though there are separate receptors for these sugars, and they suggest that the two sugar-receptor complexes compete with each other in their interaction with the next stage in the signalling system. Such competition could prevent an organism which was adequately supplied with one satisfactory carbohydrate from responding to a second, while permitting a response to a substrate with a different role, such as a nitrogen source. It seems possible that a similar system exists in myxomycetes.

Knowles \& Carlile (1978) found that on SVP agar media the lowest glucose concentration that depressed migration was $0 \cdot 1 \%(5.6 \mathrm{~mm})$ whereas the threshold for chemotaxis is about $0.25 \mathrm{~mm}$. Moreover on SVP agar at high concentrations $(56 \mathrm{~mm})$ almost all sugars, whether attractants or not, depressed the migration rate. Hence attraction by sugars cannot be related to their influence on migration rate. The osmotic effects of high sugar concentrations may, however, be responsible for both reduced migration rate (Knowles \& Carlile, 1978) and negative chemotaxis (see below). It seems likely that positive chemotactic agents act by biassing the protoplasmic shuttle streaming of plasmodia towards the point of application of the attractant (Ueda et al., 1975, 1976) by mechanisms as yet unknown.

\section{Negative chemotaxis}

Ueda et al. (1975) concluded that fructose and sucrose repelled plasmodia of $P$. polycephalum with threshold concentrations of 10 and $30 \mathrm{mM}$, respectively, and later (Ueda et al., 1976) that glucose and mannitol repelled at high concentrations (300 mm). Durham \& Ridgeway (1976) found $1 \%$ sucrose and $1 \%$ ribose to be repellents and Ludlow \& Durham (1977) reported that arabinose, glucosamine hydrochloride, $N$-acetyl-D-glucosamine and several other compounds at $1 \%$ were also repellents. In the present study all sugars and derivatives were able to repel plasmodia under appropriate conditions at $100 \mathrm{~mm}$. The hydrochlorides of amino-sugars repelled at 10 or $5 \mathrm{~mm}$; this effect is probably due to the chloride ion, since chlorides are repellents (Ueda et al., 1975).

The negative chemotaxis displayed towards sugars tested at $100 \mathrm{~mm}$ on salts agar, and many at $50 \mathrm{~mm}$ on SVP agar, may be related to the depression in migration rate caused by most sugars, whether utilizable or not, at $56 \mathrm{~mm}$ on SVP agar (Knowles \& Carlile, 1978). Both may be a consequence of exposure to high external osmotic pressures, symmetrical in the case of partial inhibition of migration, and at one end of the plasmodium in the case of negative chemotaxis. Sugars that are attractants tend to have higher thresholds (75 to $100 \mathrm{~mm}$ ) for repulsion than sugars that are not (25 to $50 \mathrm{~mm})$, an effect that is presumably due to interaction of attraction and repulsion. Differences in thresholds may also depend on the ease with which the plasmodium can adapt to changed osmotic pressures: it is possible that the high threshold for the non-attractant methyl- $\alpha$-D-glucoside may be due to more rapid penetration of the plasmodium by this compound. Glucosamine hydro- 
chloride has a very low threshold for repulsion $(5 \mathrm{~mm})$ and also inhibits migration more strongly than other compounds that have minor effects on growth rate (Knowles \& Carlile, 1978); both these effects may be due to the chloride ion.

\section{Comparison of chemotactic assay methods}

Chemotaxis is usually regarded as a response to a concentration gradient, and agar diffusion methods such as the two-well test of Carlile (1970) and the quadrant test of Madelin et al. (1975) create a gradient of the test compound. They have the disadvantage, however, that the concentration of the test compound in the well and the steepness of the gradient diminish and are unknown at the time that the response occurs. Indeed, it is likely that the gradients to which plasmodia respond in the two-well and quadrant tests are created largely by metabolism rather than by diffusion. This hypothesis is supported by the ineffectiveness of the quadrant test on water agar (only glucose and maltose attracted) as compared with SVP agar. It is probable that on water agar little utilization of the carbohydrates in test solutions will occur, since growth will be prevented by the absence of a nitrogen source, and gradients will result almost entirely from diffusion. On SVP agar, however, carbohydrate availability will be the main factor limiting growth, and rapid metabolism of sugars may be expected, leading to a steep gradient in the vicinity of the plasmodium. The failure of the quadrant test to detect attraction by 2-deoxy-D-glucose also supports the view that metabolism by the plasmodium is crucial in producing a sufficiently steep gradient, since this sugar is not metabolized and hence the gradient, which proves ineffective even on SVP agar, must result solely from diffusion. This may be the reason why Chet et al. (1977) failed to detect chemotaxis by $P$. polycephalum to 2-deoxy-D-glucose in a modified quadrant test.

A merit of the double strip test is that the opposite ends of the plasmodium are presented with a known and a zero concentration of the attractant, respectively. This is not strictly a gradient, but an organism does not respond to a gradient, but to spatial or temporal differences in concentration (Carlile, 1975). Indeed, a chemical gradient is unlikely to be more than a few $\mathrm{mm}$ in length whereas a plasmodium may be many $\mathrm{cm}$ long. Hence, in nature, a plasmodium may encounter an attractant at one point on the surface, while the opposite side is unstimulated. Therefore the double strip test may, for myxomycete plasmodia, be not only more efficient, but more realistic than agar diffusion assays.

We wish to thank the Science Research Council for a studentship to one of us (D. J.C.K.).

\section{REFERENCES}

AdLER, J. (1975). Chemotaxis in bacteria. In Primitive Sensory and Communication Systems: the Taxes and Tropisms of Micro-organisms and Cells, pp. 91-100. Edited by M. J. Carlile. London: Academic Press.

CARLILE, M. J. (1970). Nutrition and chemotaxis in the myxomycete Physarum polycephalum: the effect of carbohydrates on the plasmodium. Journal of General Microbiology 63, 221-226.

Carlile, M. J. (1972). The lethal interaction following plasmodial fusion between two strains of the myxomycete Physarum polycephalum. Journal of General Microbiology 71, 581-590.

Carlile, M. J. (1975). Taxes and tropisms: diversity, biological significance and evolution. In Primitive Sensory and Communication Systems: the Taxes and Tropisms of Micro-organisms and
Cells, pp. 1-28. Edited by M. J. Carlile. London: Academic Press.

Chet, I., Naveh, A. \& Henis, Y. (1977). Chemotaxis of Physarum polycephalum towards carbohydrates, amino acids and nucleotides. Journal of General Microbiology 102, 145-148.

Durham, A.C. H. \& Ridgeway, E. B. (1976). Control of chemotaxis in Physarum polycephalum. Journal of Cell Biology 69, 218-223.

Knowles, D. J. C. \& Carlile, M. J. (1978). Growth and migration of plasmodia of the myxomycete Physarum polycephalum: the effect of carbohydrates, including agar. Journal of General Microbiology 108, 9-15.

Ludlow, C. T. \& Durham, A. C. H. (1977). Calcium ion fluxes across the external surface of Physarum polycephalum. Protoplasma 91, 107-113. 
Madelin, M. F., Audus, F. \& Knowles, D. (1975). Attraction of plasmodia of the myxomycete, Badhamia utricularis, by extracts of the basidiomycete, Stereum hirsutum. Journal of General Microbiology 89, 229-234.

SNedecor, G. W. \& Cochran, W. G. (1974). Statistical Methods, 6th edn. Ames, Iowa: Iowa State University Press.

Strange, P. G. \& Koshland, D. E. (1976). Receptor interactions in a signalling system: competition between galactose receptor and ribose receptor in the chemotaxis response. Proceedings of the National Academy of Sciences of the United States of America 73, 762-766.
Sweeley, C. C., Bentley, R., Makita, M. \& Wells, W. W. (1963). Gas-liquid chromatography of trimethylsilyl derivatives of sugars and related substances. Journal of the American Chemical Society 85, 2497-2507.

Ueda, T., Terayama, K., Kurihara, K. \& KobaTAKE, Y. (1975). Threshold phenomena in chemoreception and taxis in the slime mould Physarum polycephalum. Journal of General Physiology 65, 223-234.

Ueda, T., Muratsugu, M., Kurihara, K. \& Kobatake, Y. (1976). Chemotaxis in Physarum polycephalum. Experimental Cell Research 100, 337-344. 